\title{
Effect of COVID-19 Pandemic on Anti-
} VEGF treatment of Medical Retinal

\section{Conditions - An Audit}

\section{Abstract}

Introduction: Ophthalmology services have been significantly impacted by the COVID-19 pandemic. Frequency of intravitreal anti-vascular endothelial growth factor (Anti-VEGF) injections are important in visual outcomes.

Methods: We conducted an audit on intravitreal services in a NHS district general hospital in the UK including all new patients with diabetic macular oedema (Cl-DMO) and wet age-related macular degeneration (AMD) who were initiated on intravitreal injection of Aflibercept (EYLEA) between $1^{\text {st }}$ January to $15^{\text {th }}$ July 2020, and had subsequent injections until October 2020. Data on injection dates and visual acuity was extracted, and the total number of all intravitreal injections for all indications between January to September 2020 and the same period in 2019. Delay to treatment was defined as more than 14 days, according to the fixed dosing schedule.

Results: We found $31 \%(n=17)$ of patients initiated on treatment for wet $\mathrm{AMD}$ and $44 \%(\mathrm{~N}=11)$ for $\mathrm{Cl}-\mathrm{DMO}$ had delayed injections. There was no correlation between total duration of delay and change in best corrected visual acuity (BCVA). Similarly, we found no association between duration of delay and change in BCVA. The number of intravitreal injections declined during the COVID19 pandemic by $17.8 \%$ compared to 2019 .

Conclusion: Majority of patients initiated on anti-VEGF injections just before the pandemic or during the pandemic received injections on time. Where there were significant delays to treatment, there was no detected loss in vision over the short term. However, the long-term impact and impact of overall reduction in intravitreal injections are unknown.

Keywords

Anti-vascular endothelial growth factor injections, intra-vitreal services, COVID-19 pandemic

\section{Introduction:}

The coronavirus disease 2019 (COVID-19) global pandemic affected the UK in March 2020. All routine hospital appointments and treatments were cancelled to prioritise resources towards treating COVID-19

\author{
Mana Rahimzadeh, Ramu \\ Muniraju \& Shahrnaz Izadi \\ Epsom and St Helier University \\ Hospitals, London, UK
}

Mana.rahimzadeh@gmail.com

Cite as: Rahimzadeh, M., Muniraju, R., Izadi, S. (2021) Effect of COVID-19 pandemic on anti-VEGF treatment of medical retinal conditions. The Physician vol 6; issue 3: 1-10 ePub 28.12.2020 DOI: https://doi.org/10.38192/1.6.3.10

Article Information

Submitted 17.12.2020

Published 28.12.2020

Creative Commons Licence

International derivative v4.0 infected patients. This significantly impacted practice in ophthalmology, dramatically reducing capacity within the hospital eye services ${ }^{1}$. It has been paramount to weigh the risk of acquiring COVID-19 against the risk of coming to harm through failure to treat serious eye disease. Thus, for many patients, 
hospital visits have been postponed to reduce exposure to the virus.

Anti-vascular endothelial growth factor (Anti-VEGF) intravitreal therapy has revolutionised the treatment of common causes of blindness including wet agerelated macular degeneration (AMD) and centre involving diabetic macular oedema (Cl-DMO). Notably, the frequency of intravitreal anti-VEGF injections has been shown to be an important contributing factor in improving and maintaining visual outcomes ${ }^{2-5}$ and delays have been shown to be associated with decreased vision ${ }^{6}$. The current National Institute for Health and Care Excellence (NICE) recommendations for posology of aflibercept (EYLEA) are:

1. Wet AMD: one treatment per month for three consecutive doses, followed by one injection every two months up to twelve months $^{7}$

2. Cl-DMO: one treatment per month for five consecutive doses, followed by one injection every two months up to twelve months ${ }^{8}$

At our unit, we use a fixed regimen treatment protocol during year one, rather than a treat and extend (T\&E). Therefore, during year one, wet AMD patients receive one injection per month for three consecutive doses followed by fixed two monthly injections up to twelve months, giving a total of seven injections. Using a T\&E dosing regimen for wet $A M D$, patients also receive one injection per month for three consecutive doses then the treatment interval is extended to two months. However, based on the physician's judgement, the treatment interval may be maintained at two months or further extended according to visual and/or anatomic outcomes ${ }^{9}$.

In response to the COVID-19 pandemic, the Royal College of Ophthalmologists (RCOphth) published guidance to aid resource prioritisation and risk stratification $^{10,11}$. Regarding new patients, their guidance was to continue intravitreal injection (IVI) treatment for wet AMD (three monthly loading doses then fixed bimonthly IVI in year one). However, they advised that IVI treatment for patients with $\mathrm{CI}-\mathrm{DMO}$ could be deferred by four to six months.

At our unit, we followed the RCOphth guidance with regards to wet AMD. However, given that our eye unit has a smaller cohort of $\mathrm{Cl}$-DMO patients compared to other larger centres, the decision was taken to continue to offer patients treatment provided they were happy to attend and understood the risks of
COVID-19 transmission. Therefore, instead of deferring treatment, we continued to offer five loading doses followed by fixed bimonthly doses in year one and telephone consultations in year two and beyond.

The aim of this retrospective audit was to evaluate the impact of COVID-19 on IVI services at our unit; specifically, to assess whether patients who had IVI treatment initiated just before or during the pandemic had any delays to their fixed dosing treatment schedule. Where significant delays to treatment resulted, we evaluate whether there was any resulting loss in visual acuity. We also compare the total number of IVI for all indications in the period January to September 2020 to the equivalent period in 2019 to assess the overall impact of COVID-19 on the service.

\section{Methods:}

We conducted an Electronic Medical Records (EMR) audit evaluating the effects of the COVID-19 pandemic on intravitreal services at an NHS district general hospital in the UK. We used Medisoft audit suite tool to extract all patients who had initial (baseline) IVI treatment of wet AMD or Cl-DMO with EYLEA between $1^{\text {st }}$ January to $15^{\text {th }}$ July 2020 and had subsequent follow up or injections until October 2020.

Data was manually extracted from Medisoft EMR for all visits up to $8^{\text {th }}$ October 2020, when analysis was commenced. Data collected included dates of IVI and best corrected visual acuity (BCVA) which was documented in the form of ETDRS (Early Treatment Diabetic Retinopathy Study) letter score. We also extracted total number of all IVI for all indications at our unit between January-September 2020 and the same period in 2019 for comparison.

Delay to treatment was defined as IVI given more than 14 days after it was scheduled to be given according to fixed dosing schedule. A delay of less than 14 days is not uncommon in clinical practice given the twostop nature of the service.

P Values were calculated using Mann Whitney U Test to evaluate the statistical significance of the effect of delay on overall change in BCVA. Linear regression analysis was conducted to evaluate the relationship between delays to treatment and BCVA.

\section{Results:}

There were a total of 82 patients ( 88 eyes) of which 2 patients had deceased within 2 months of initiating treatment and were therefore excluded from analysis. 
Of these, 55 patients (57 eyes) were treated for wet AMD and 25 patients ( 29 eyes) for Cl-DMO.

We found that $69 \%$ of patients $(n=38)$ receiving their first IVI for wet AMD between $1^{\text {st }}$ January and $15^{\text {th }}$ July 2020 had timely treatments whilst $31 \%(n=17)$ had a delay of more than 14 days from the fixed dosing schedule. Meanwhile, 56\% $(n=14)$ of patients receiving their first IVI for $\mathrm{Cl}-\mathrm{DMO}$ during this same period received timely treatment and $44 \%(\mathrm{~N}=11)$ had a delay of more than 14 days from the fixed dosing schedule (Fig.1).

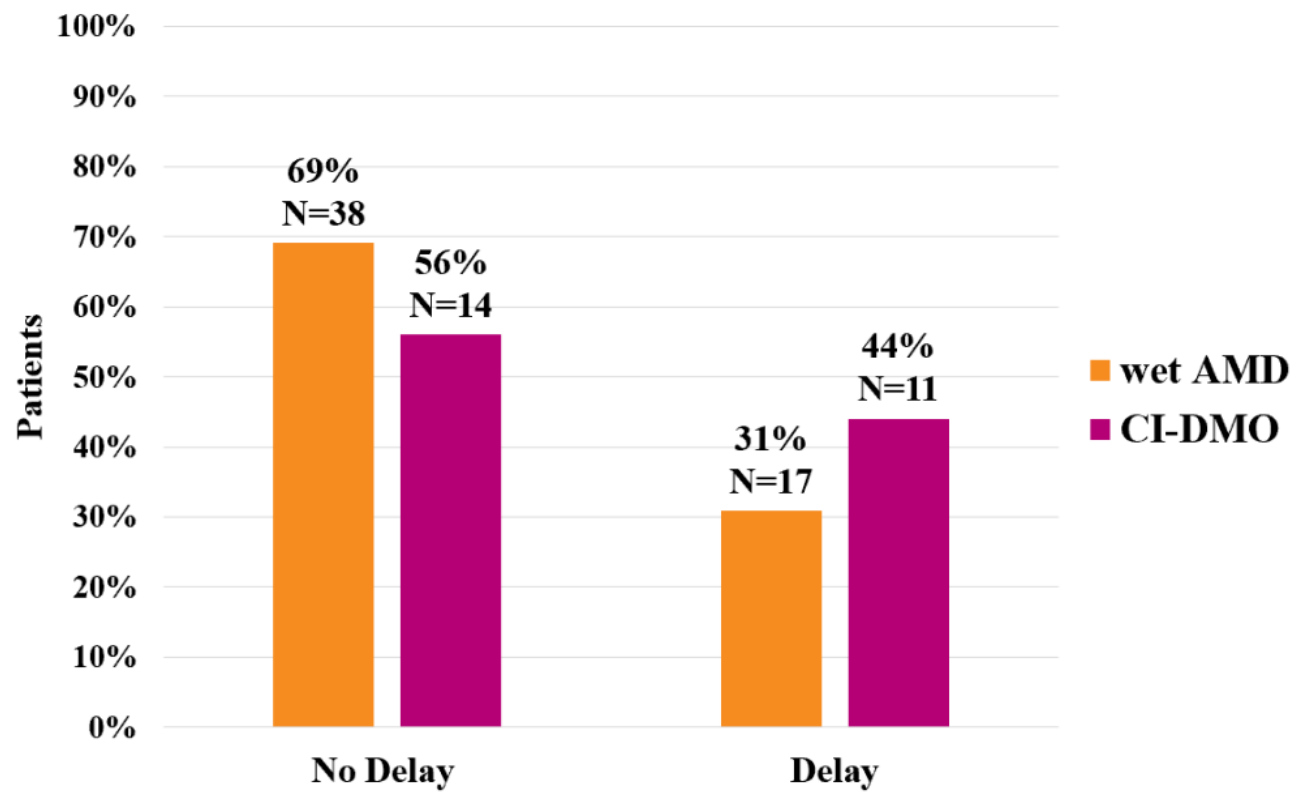

Figure 1. A graph showing the percentage of patients who had a delay in treatment according to indication

Figure 2 illustrates the overall change in BCVA (most recent BCVA letter score minus baseline BCVA letter score) stratified according to whether there was a delay in treatment of the eye. In wet AMD, the mean change in BCVA (dottedline) was more negative for those with a delay to treatment $(-1.33)$ than those without (+4.41) and the median change in BCVA was 0 and +5 respectively $(P=0.19)$. In $\mathrm{Cl}-\mathrm{DMO}$, the mean change in BCVA was more positive for those with a delay to treatment $(+3.38)$ than those without $(-2.50)$ and median change was +5 in both groups $(P=0.51)$. 

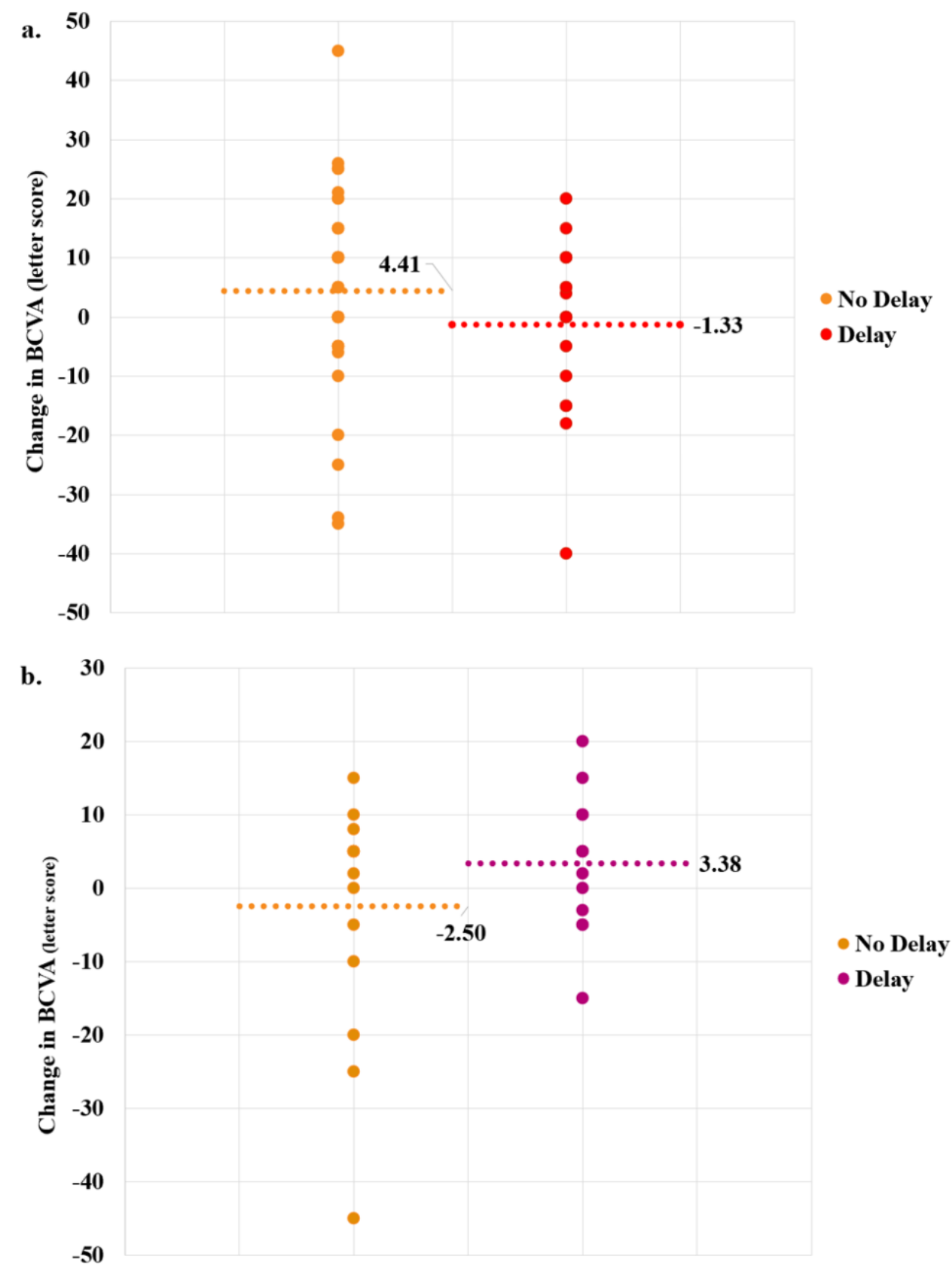

Figure 2. Graphs showing the overall change in BCVA stratified according to delay in treatment with EYLEA IVI for a) wet AMD: no delay mean 4.41, median 5, delay mean 1.33, median 0 ( $p=0.19$ ) b) Cl-DMO: no delay mean -2.50 , median 5 , delay mean 3.38 , median $5(p=0.51)$

A total of 17 patients with wet $A M D$ and 11 patients with $\mathrm{Cl}-\mathrm{DMO}$ had a delay to treatment from the fixed dosing schedule. Of these, 5 patients with wet AMD and 3 patients with $\mathrm{Cl}-\mathrm{DMO}$ had more than one delay period during their treatment. The mean, median and range of delays from the scheduled IVI treatment date are summarised in Table 1 . Of those patients who had a delay to their treatment, $82 \%$ of these delays ( $\mathrm{N}=14$ wet $\mathrm{AMD}$ and $\mathrm{N}=9 \mathrm{Cl}-\mathrm{DMO}$ ) were during the COVID-19 period (defined as $15^{\text {th }}$ March to $15^{\text {th }}$ July when there was the greatest disruption to services). There was no significant relationship between total delay (sum of individual delays per eye throughout the treatment course) and overall change in BCVA for either indication as shown by $\mathrm{R}^{2}$ values (Figure 3). 


\begin{tabular}{|lll|}
\hline Delay (days) & AMD & DMO \\
\hline Mean & 47 & 56 \\
\hline Median & 26 & 31 \\
\hline Range & $15-166$ & $17-207$ \\
\hline
\end{tabular}

Wet AMD: 17 patients

CI-DMO: 11 patients

Table 1: Table summarising the mean, median and range of delay from the fixed dosing schedule in days
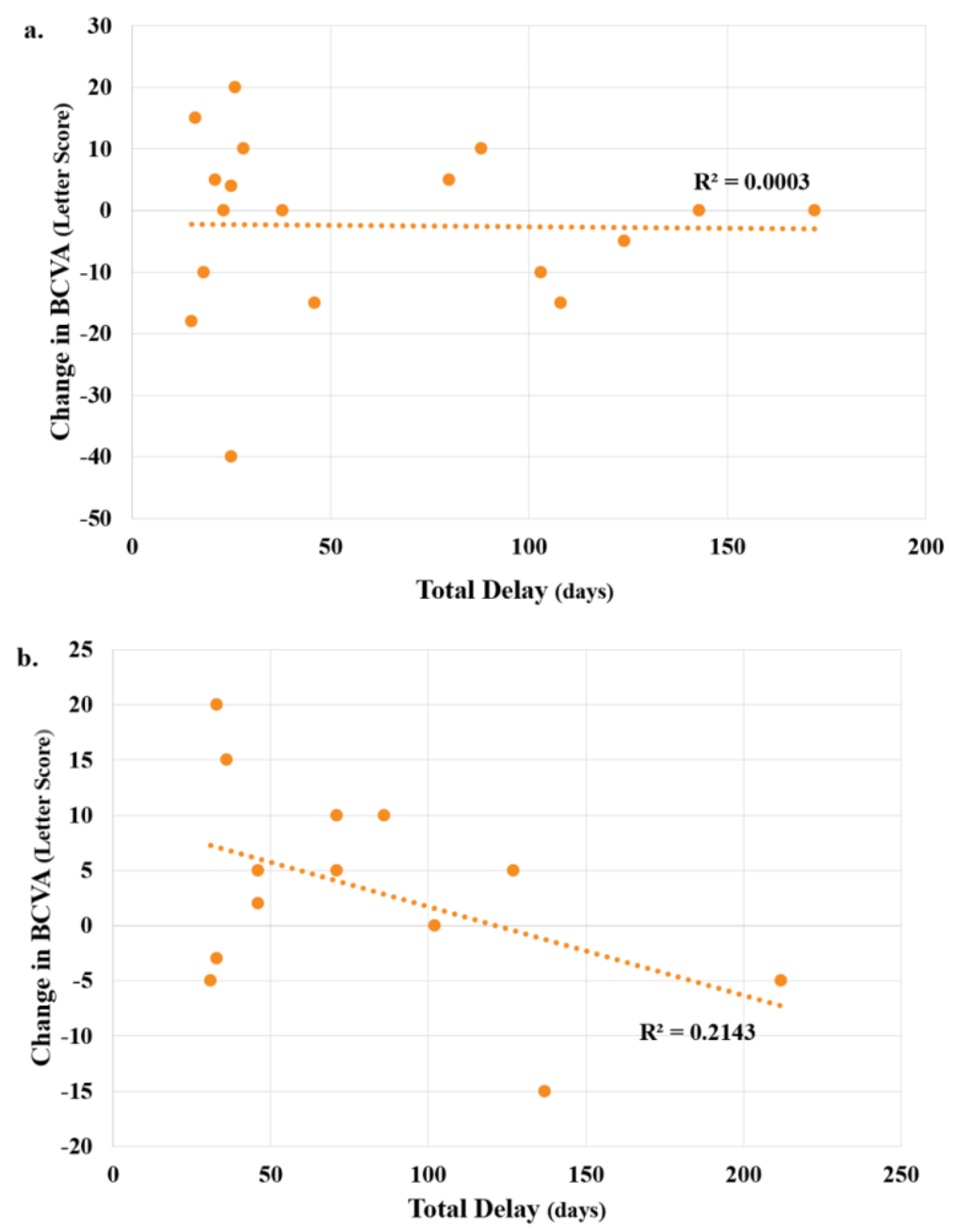

Figure 3. Relationship between total delay and overall change in BCVA in a. wet $A M D\left(R^{2}=0\right)$ and $b$. Cl-DMO $\left(R^{2}=0.21\right.$, entirely dependent on data points at 137 days and 212 days $)$ 
Furthermore, there was no significant correlation between duration of delay in treatment between injections and change in BCVA between those same treatments as shown by the $R^{2}$ values of the linear regression models (Fig. 4). For AMD the change in treatment regimen from monthly to 8-weekly was considered in the analysis (Fig 4). For $\mathrm{Cl}-\mathrm{DMO}$, there were no patients with delay in their treatment who had received more than the first five monthly injections therefore no change in treatment regimen was considered in analysis.
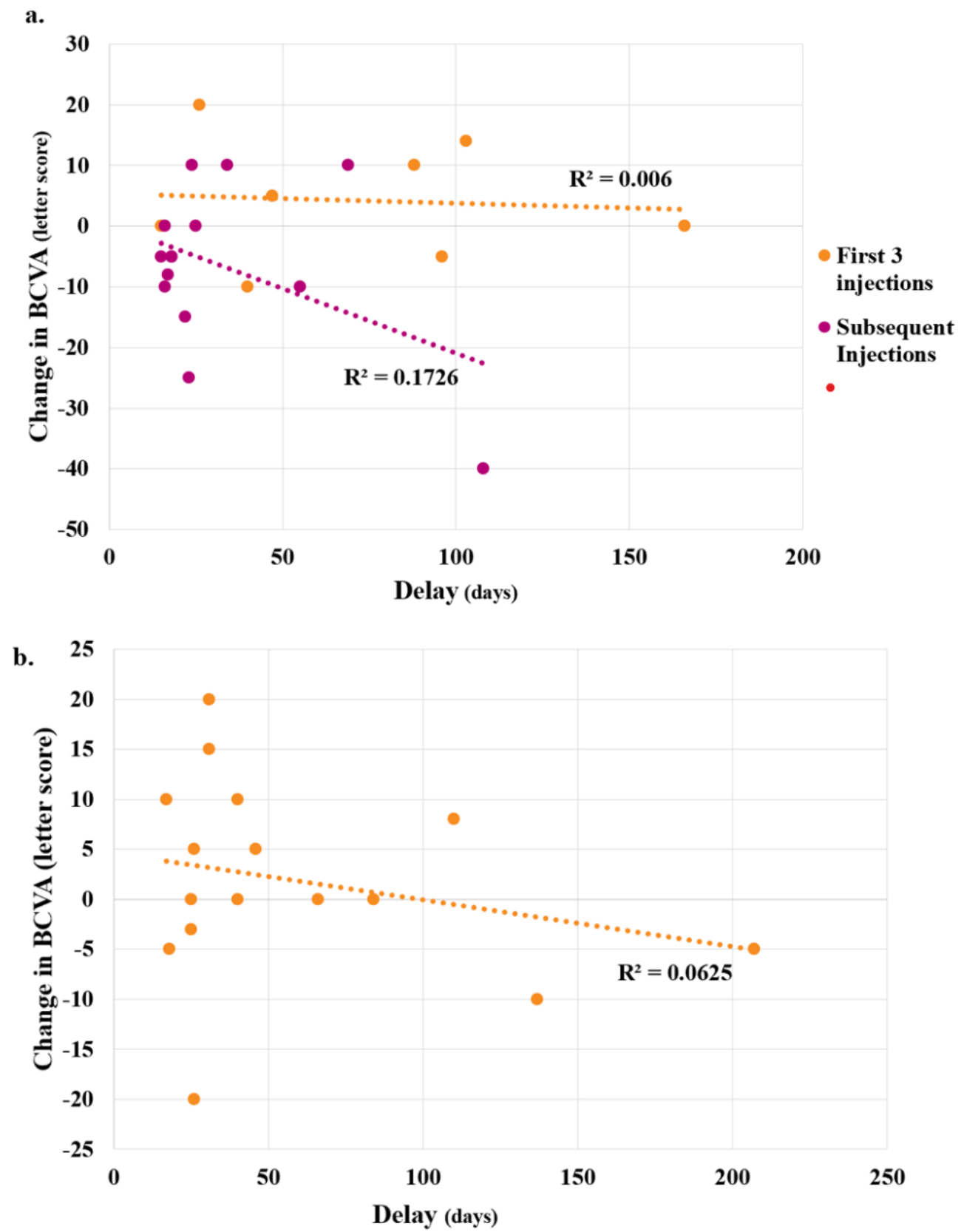

Figure 4. Relationship between delay in treatment and change in BCVA between these delayed treatments in a. AMD stratified according to first 3 monthly injections $\left(R^{2}=0\right)$ and subsequent 8 weekly injections $\left(R^{2}=0.17\right.$, entirely dependent on single point at 105 days) and b. Cl-DMO first 5 monthly injections only $\left(\mathrm{R}^{2}=0.06\right)$ 
Analysis of total injections for all indications between January-September 2020 compared to the same period in 2019 showed an overall decrease by $17.8 \%$ in injections at this eye unit. Fig. 5).

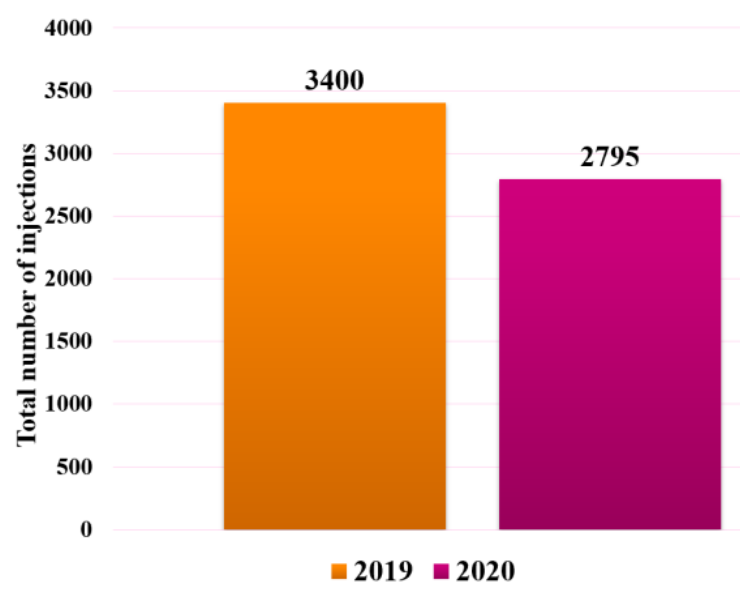

Figure 5. Comparison of the total number of anti-VEGF injections in the same period in 2019 and 2020 for all indications

\section{Discussion:}

Our results suggest that during the COVID-19 period the majority of wet AMD patients and many Cl-DMO patients initiated on IVI treatment at our unit received treatments on time. However, there were fewer overall IVI at the department compared to same period in 2019, including new and follow up patients.

We found no significant correlation between the total duration of delay and overall change in BCVA over the treatment period. We also found no association between treatment delay between subsequent injections and change in BCVA between these same treatments.

There was marginal loss of vision $(p=0.19)$ in wet AMD due to delay, however $\mathrm{Cl}$-DMO patients who had a delay to treatment had a relative improvement in BCVA ( $p=0.50)$. In both cases, these changes in BCVA were not statistically significant. However, it is plausible that delay in treatment of $\mathrm{Cl}$-DMO may not significantly affect BCVA due to the more indolent nature of maculopathy. Delay in treatment was also found not to affect final BCVA outcomes in the recent DRCR.net Protocol V study ${ }^{12}$.

The approach to the pandemic has been variable, with some eye units maintaining IVI injections even through the height of the pandemic ${ }^{13}$ and other units experiencing a significant drop in their IVI services ${ }^{14,15}$. There is still limited literature evaluating the impact of delays to IVI treatment during COVID-19 on visual outcomes and to our knowledge, there is no study looking at patients initiated on IVI treatment during or just prior to the pandemic.

One study in Northern Italy evaluated the impact of delays secondary to COVID-19 on maintenance IVI treatment of wet AMD. They found a significant association between interval time between treatments and BCVA in wet AMD patients who were established on a PRN approach prior to the pandemic $^{16}$.

Given that the COVID-19 pandemic and its impact on ophthalmology services is ongoing, it is important to evaluate the risk of short- and long-term vision loss associated with extending intervals between treatments against the prevalence of COVID-19 in community and individual patient comorbidities.

\section{Limitations and considerations:}

Our analysis of the effect of delays in IVI treatment on BCVA was limited as the majority of our patients did not have a delay to treatment. Furthermore, our follow up was limited to nine months, but it would be important to consider the longer-term effects of delays to IVI treatment. We therefore intend to conduct a follow up study to assess the longer-term effects of these delays on visual outcomes of these patients.

\section{References:}


1. Safadi K, Kruger JM, Chowers I, et al. Ophthalmology practice during the COVID-19 pandemic. BMJ open ophthalmology. 2020;5(1):e000487-e000487. doi:10.1136/bmjophth-2020-000487

2. Oubraham $H$, Cohen SY, Samimi S, et al. INJECT AND EXTEND DOSING VERSUS DOSING AS NEEDED: A Comparative Retrospective Study of Ranibizumab in Exudative Age-Related Macular Degeneration. RETINA. 2011;31(1). doi:10.1097/IAE.0b013e3181de5609

3. Lee R, Wong TY, Sabanayagam C. Epidemiology of diabetic retinopathy, diabetic macular edema and related vision loss. Eye and vision (London, England). 2015;2:17. doi:10.1186/s40662-015-0026-2

4. Holz FG, Tadayoni R, Beatty $S$, et al. Multicountry real-life experience of anti-vascular endothelial growth factor therapy for wet agerelated macular degeneration. British Journal of Ophthalmology. 2015;99(2):220. doi:10.1136/bjophthalmol-2014-305327

5. Lanzetta $P$, Loewenstein A, Committee VAS. Fundamental principles of an anti-VEGF treatment regimen: optimal application of intravitreal anti-vascular endothelial growth factor therapy of macular diseases. Graefe's archive for clinical and experimental ophthalmology. 2017;255(7):1259-1273. doi:10.1007/s00417-017-3647-4

6. Massamba N, Dirani A, Knoeri J, Pasquier B, Ingram $A$, Soubrane $G$. Evaluating the impact of summer vacation on the visual acuity of AMD patients treated with ranibizumab. Eye. 2015;29(11):1453-1457.

doi:10.1038/eye.2015.128

7. National Institute for Health and Care Excellence. Aflibercept solution for injection for treating wet age-related macular degeneration [Internet]. [London]: NICE; 2013. Available from: nice.org.uk/guidance/ta294

8. National Institute for Health and Care Excellence. Aflibercept for treating diabetic macular oedema. [Internet]. [London]: NICE; 2013. Available from: nice.org.uk/guidance/ta346
9. Ross $\mathrm{AH}$, Downey $\mathrm{L}$, Devonport $\mathrm{H}$, et al. Recommendations by a UK expert panel on an aflibercept treat-and-extend pathway for the treatment of neovascular age-related macular degeneration. Eye (Basingstoke). 2020;34(10):1825-1834. doi:10.1038/s41433019-0747-x

10. Royal College of Ophthalmologists. Medical Retinal Management Plans during COVID-19. [Internet]. [London]: RCOPhth; March 2020. Available from: https://www.rcophth.ac.uk/wpcontent/uploads/2020/10/Medical-RetinalManagement-Plan-During-COVID-19.pdf

11. Royal College of Ophthalmologists. Medical Retinal Management Plans during COVID-19. [Internet]. [London]: RCOPhth; September 2020. Available from: https://www.rcophth.ac.uk/wpcontent/uploads/2020/03/Medical-RetinalManagement-Plan-during-COVID-19UPDATED-300320-1-3.pdf

12. Baker CW, Glassman AR, Beaulieu WT, et al. Effect of Initial Management With Aflibercept vs Laser Photocoagulation vs Observation on Vision Loss Among Patients With Diabetic Macular Edema Involving the Center of the Macula and Good Visual Acuity: A Randomized Clinical Trial. JAMA. 2019;321(19):1880-1894. doi:10.1001/jama.2019.5790

13. Campos A, Oliveira N, Martins J, Arruda H, Sousa J. The Paradigm Shift of Ophthalmology in the COVID-19 Era. Clin Ophthalmol. 2020;14:2625-2630 https://doi.org/10.2147/OPTH.S267427

14. Wasser LM, Weill Y, Brosh K, et al. The Impact of COVID-19 on Intravitreal Injection Compliance [published online ahead of print, 2020 Oct 28]. SN Compr Clin Med. 2020;1-4. doi:10.1007/s42399-020-00614-4

15. Borrelli E, Grosso D, Vella G, et al. Impact of COVID-19 on outpatient visits and intravitreal treatments in a referral retina unit: let's be ready for a plausible "rebound effect". Graefes Arch Clin Exp Ophthalmol. 2020;258(12):2655-2660. doi:10.1007/s00417-020-04858-7 
16. Borrelli E, Grosso D, Vella G, et al. Short-term outcomes of patients with neovascular exudative AMD: the effect of COVID-19 pandemic. Graefes Arch Clin Exp Ophthalmol. 2020;258(12):2621-2628.

doi:10.1007/s00417-020-04955-7

\section{Conflicts of interest:}

Dr Mana Rahimzadeh, Foundation Year Two Doctor: No conflicts of interest
Mr Ramu Muniraju, Consultant Ophthalmologist with special interest in medical retina: Received travel grants and hospitality to attend educational meetings from Allergan and Bayer

Miss Shahrnaz Izadi, Consultant Ophthalmologist with a special interest in medical retina: Received travel grants and hospitality to attend educational meetings from Novartis 